\title{
Electronic Tuning of Misaligned Coils In Wireless Power Transfer Systems
}

\author{
Samer Aldhaher, Patrick C. K. Luk, Senior Member, IEEE James F. Whidborne, Senior Member, IEEE \\ School of Engineering, Cranfield University, Bedford, UK \\ p.c.k.luk@cranfield.ac.uk
}

\begin{abstract}
The misalignment and displacement of inductively coupled coils in a wireless power transfer system (WPT) can degrade the power efficiency and limit the amount of power that can be transferred. Coil misalignment leads the primary coil driver to operate in an untuned state which causes non-optimum switching operation and results in an increase in switching losses. This paper presents a novel method to electronically tune a Class $E$ inverter used as a primary coil driver in an inductive WPT system to minimize the detrimental effects of misalignment between the inductively coupled coils which may occur during operation. The tuning method uses current controlled inductors (saturable reactors) and a variable switching frequency to achieve optimum switching conditions regardless of the misalignment. Mathematical analysis is performed on a Class $\mathbf{E}$ inverter based on an improved model of a resonant inductive link. Experimental results are presented to confirm the analysis approach and the suitability of the proposed tuning method.
\end{abstract}

Index Terms-Inductive power transmission, Coupling circuits, Resonant inverters, Tunable circuits and devices

\section{INTRODUCTION}

Research in wireless power transfer (WPT) technologies based on magnetic induction is gaining momentum recently due to the increasing reliance on battery-powered applications ranging from electric vehicles to mobile devices and medical implants [1]-[6]. However, the issues of coil misalignment and displacement, highlighted in wireless charging of electric vehicles, appear to be not adequately addressed. Improper parking of the vehicle will result in the wireless charging platform operating at a degraded performance of lower efficiency, reduced power transfer and longer charging time. A possible solution is to include additional coils either in the transmitter or in the vehicle so that the effect of misalignment is reduced over a certain range [2], [7]-[9]. The main disadvantages of this solution are larger required surface area, higher cost and weight of the overall system. An alternative solution that addresses these shortcomings is to retune the transmitter in order to operate at optimum switching conditions when the coils are in a misaligned state [5], [10]-[15]. This paper further explores and investigates the viability and practicality of the tuning solution presented in [10], [11] by using saturable reactors to tune a Class $\mathrm{E}$ DC/AC inverter designed to drive an inductive link at $800 \mathrm{kHz}$.

This paper is organised as follows. Section II derives analytical expressions of the reflected impedances of a resonant inductive link while considering the equivalent series resistance (ESR) of the resonant elements. Section III analyses the Class E inverter including the reflected impedances of the

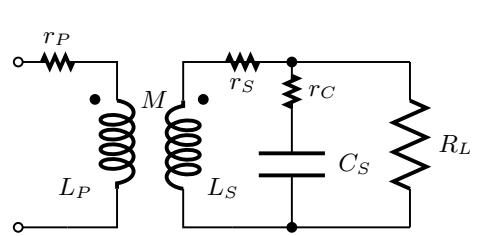

(a) circuit representation

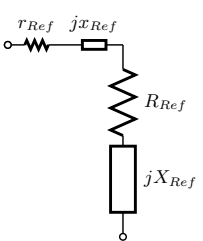

(b) equivalent circuit
Fig. 1. Resonant inductive link circuit model

inductive link. A numerical solution is presented to calculate the values of the Class E inverter's parameters to be controlled as the coupling coefficient of the inductive link varies. Section IV discusses the use of saturable reactors as tuning elements and the key design aspects. Section V describes an implemented WPT system, the principle of operation of the electronic tuning method is discussed and extensive results based on experimental measurements are presented. Finally, Section VI includes the conclusion and future work.

\section{INDUCTIVE LINK MODEL}

Fig. 1a shows a typical inductively coupled circuit, which consists of a primary coil represented by inductance $L_{P}$ and its ESR $r_{P}$, and a secondary coil represented by an inductance $L_{S}$ and its ESR $r_{S}$. The ESR of the primary and the secondary coils includes the ohmic resistance, the skin effect and the proximity effect. A capacitor $C_{S}$ is connected in parallel with the secondary coil to allow for resonant operation. Resistance $r_{C}$ represents the ESR of the capacitor which includes its dielectric losses. The load represented by a resistance $R_{L}$ is connected in parallel with the secondary coil. Inductance $M$ represents the mutual inductance between the primary and secondary coils and is equal to

$$
M=k \sqrt{L_{P} L_{S}}
$$

where $k$ is the coupling coefficient between the primary and secondary coils.

The reflected impedance of the secondary coil seen by a voltage source connected to the primary coil is 


$$
\begin{gathered}
Z_{\text {Ref }}=\frac{X_{M}^{2}}{r_{S}+j X_{L_{S}}+\frac{R_{L}\left(r_{C_{S}}-j X_{C_{S}}\right)}{R_{L}+r_{C_{S}}-j X_{C_{S}}}} \\
=\frac{X_{M}^{2}\left(R_{L} a+r_{S} a-j X_{C_{S}} a-j R_{L} b-j r_{C_{S}} b-X_{C_{S}} b\right)}{a^{2}+b^{2}}
\end{gathered}
$$

where $X_{L_{P}}, X_{L_{S}}, X_{M}$ and $X_{C}$ are the reactances of the primary coil, the secondary coil, the mutual inductance and the parallel capacitor respectively, and

$$
\begin{aligned}
a & =R_{L}\left(r_{S}+r_{C_{S}}\right)+r_{S} r_{C_{S}}+X_{L_{S}} X_{C_{S}} \\
b & =R_{L}\left(X_{L_{S}}-X_{C_{S}}\right)+X_{L_{S}} r_{C_{S}}-X_{C_{S}} r_{S} .
\end{aligned}
$$

The reflected impedance of the secondary coil circuitry can be written in the form

$$
Z_{\mathrm{Ref}}=R_{\mathrm{Ref}}+r_{\mathrm{Ref}}+j X_{\mathrm{Ref}}+j x_{\mathrm{Ref}}
$$

where $R_{\text {Ref }}$ is the real part of the reflected impedance corresponding to the load and is equal to

$$
R_{\mathrm{Ref}}=\frac{X_{M}^{2} X_{C_{S}}^{2} R_{L}}{a^{2}+b^{2}}
$$

Resistance $r_{\text {Ref }}$ is the real part of the reflected impedance corresponding to the ESR of the coil and the capacitor and is equal to

$$
\begin{gathered}
r_{\mathrm{Ref}}= \\
\frac{X_{M}^{2}\left(R_{L}^{2}\left(r_{S}+r_{C_{S}}\right)+2 R_{L} r_{S} r_{C_{S}}+R_{L} r_{C_{S}}^{2}+r_{S} r_{C_{S}}^{2}+X_{C_{S}}^{2} r_{S}\right.}{a^{2}+b^{2}}
\end{gathered}
$$

Reactance $X_{\text {Ref }}$ is the imaginary part of the reflected impedance which corresponds to the coil, the parallel capacitor and load resistance and is equal to

$$
X_{\text {Ref }}=\frac{X_{M}^{2}\left(R_{L}^{2}\left(X_{C_{S}}-X_{L_{S}}\right)-X_{L_{S}} X_{C_{S}}^{2}\right)}{a^{2}+b^{2}} .
$$

and $x_{\text {Ref }}$ is the imaginary part of the reflected impedance corresponding to the ESR of the coil and the capacitor and is equal to

$$
x_{\text {Ref }}=\frac{X_{M}^{2}\left(2 X_{C_{S}} R_{L} r_{C_{S}}-X_{L_{S}} r_{C_{S}}^{2}\right)}{a^{2}+b^{2}} .
$$

Equations 6-9 can be represented by the equivalent circuit shown in Fig. 1b. The equivalent circuit of the inductive link is used in the analysis of the primary coil driver in the following section.

\section{Class E Inverter with Finite DC-FeEd INDUCTANCE}

\section{A. Analysis}

A DC/AC inverter is required to drive the primary coil of the inductive link with a high frequency current. Different topologies and classes are available that can meet the requirements of inductive links. The Class E inverter, invented by the Sokals in 1975 [16], is a certain class of amplifiers that has been widely used recently as the primary coil driver in resonant inductive links due to its simplicity, high efficiency and high power delivering capability [5], [17]-[24]. Detailed description and

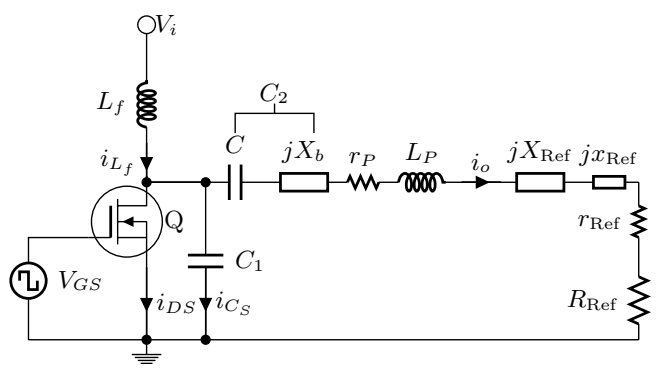

Fig. 2. Class E inverter circuit including the reflected impedances of the inductive link

analysis of Class $\mathrm{E}$ inverters or amplifiers are well documented in literature for a fixed load resistance [25]-[32]. The analysis of the Class E inverter presented in this paper takes into consideration the reflected impedance of the inductive link. The circuit diagram of a Class E inverter including the reflected impedances of the coupled coils is shown in Fig. 2. Inductance $L_{f}$ is referred to as the finite DC-feed inductance, capacitance $C_{1}$ is a shunt capacitor connected across MOSFET $Q$. The output load network consists of the primary coil inductance $L_{P}$ and its ESR, the reflected impedances of the secondary coil at a certain coupling coefficient and a residual impedance $X_{b}$. Capacitance $C$, the primary coil inductance and the imaginary components of the reflected impedance are tuned to the switching frequency. In general, the residual impedance )may or may not be a physical component. It is assumed in this paper that the residual impedance and capacitance $C$ form a physical capacitor and is referred to as $C_{2}$.

Tuning will be achieved by varying the value of the DCfeed inductance and the switching frequency as the coupling coefficient between the coils varies. Therefore the main aim of the analysis is to derive a relationship between the reflected impedances and the values of the DC-feed inductance and the switching frequency required to achieve the optimal switching conditions of the Class E inverter. The analysis is based on the following assumptions:

- The MOSFET is an ideal switch whose ON resistance is zero, and its turn on and off times are zero.

- The loaded quality factor of the series load network is sufficiently high enough such that the current flowing through it $\left(i_{o}\right)$ is sinusoidal and is equal to

$$
i_{o}(\omega t)=i_{m} \sin (\omega t+\phi) .
$$

- The duty cycle of the MOSFET gate drive signal is $50 \%$. Applying KCL and KVL at the MOSFET's drain node results in the following two equations

$$
\begin{array}{r}
i_{L_{f}}(\omega t)=i_{D S}(\omega t)+i_{C_{1}}(\omega t)+i_{o}(\omega t) \\
V_{i}-v_{D S}(\omega t)=v_{L_{f}}(\omega t)=\omega L_{f} \frac{d i_{L_{f}}(\omega t)}{d \omega t} .
\end{array}
$$

For the period $0 \leq \omega t<\pi$, the MOSFET is ON, therefore

$$
\begin{aligned}
v_{D S}(\omega t) & =0 \\
i_{C_{1}}(\omega t) & =0 \\
v_{L_{f}}(\omega t)=V_{i} & =\omega L_{f} \frac{d i_{L_{f}}(\omega t)}{\omega t} .
\end{aligned}
$$


The DC-feed inductor's current is

$$
i_{L_{f}}(\omega t)=\frac{1}{\omega L_{f}} \int_{0}^{\omega t} V_{i} d \omega t+i_{L_{f}}(0)=\frac{V_{i}}{\omega L_{f}} \omega t+i_{L_{f}}(0) .
$$

The MOSFET's drain current is given by

$$
i_{D S}(\omega t)=i_{L_{f}}(\omega t)-i_{o}(\omega t)=I_{L_{f}}(\omega t)-i_{m} \sin (\omega t+\phi) .
$$

Since the MOSFET's drain current is zero before it is switched ON, i.e

$$
i_{D S}(0)=i_{L_{f}}(0)-i_{m} \sin \phi
$$

therefore,

$$
i_{L_{f}}(0)=i_{m} \sin \phi
$$

For the period $\pi \leq \omega t<2 \pi$, the MOSFET is switched OFF, therefore

$$
\begin{aligned}
& i_{D S}(\omega t)=0 \\
& v_{D S}(\omega t)=v_{C_{1}}(\omega t) .
\end{aligned}
$$

The voltage across the DC-feed inductance is

$$
v_{L_{f}}(\omega t)=V_{i}-v_{D S}=\omega L \frac{d i_{L_{f}}(\omega t)}{d \omega t} .
$$

The current through the shunt capacitor can be determined by differentiating Eq. 22

$$
i_{C_{1}}(\omega t)=-\omega C_{1} \frac{d V_{D S}(\omega t)}{d \omega t}=\omega^{2} L_{f} C_{1} \frac{d^{2} i_{L_{f}}(\omega t)}{d \omega t^{2}} .
$$

According to Eq. 11, the DC-feed inductor's current is

$$
\begin{aligned}
i_{L_{f}}(\omega t) & =i_{C_{1}}(\omega t)+i_{o}(\omega t) \\
& =i_{m} \sin (\omega t+\phi)-\omega^{2} L_{f} C_{1} \frac{d^{2} i_{L_{f}}(\omega t)}{d \omega t^{2}} .
\end{aligned}
$$

Rearranging the above equation and normalising with respect to $i_{m}$ gives

$$
\omega^{2} L_{f} C_{1} \frac{d^{2} \frac{i_{L_{f}}}{i_{m}}(\omega t)}{d \omega t^{2}}+\frac{i_{L_{f}}}{i_{m}}(\omega t)-\sin (\omega t+\phi)=0 .
$$

Equation 25 is a linear non-homogeneous second-order differential equation which has the following general solution

$\frac{i_{L_{f}}}{i_{m}}(\omega t)=A \sin (q \omega t)+B \cos (q \omega t)+\frac{q^{2} \sin (\omega t+\phi)}{q^{2}-1}, \quad q \neq 1$

where

$$
q=\frac{1}{\omega \sqrt{L_{f} C_{1}}}
$$

and the coefficients $A$ and $B$ are determined from the continuity conditions of the DC-feed inductor's current and the shunt capacitor's voltage during the transition from the ON state to the OFF state, i.e

$$
i_{L_{f}}\left(\pi^{+}\right)=i_{L_{f}}\left(\pi^{-}\right)=\frac{V_{i} \pi}{\omega L_{f}}+i_{m} \sin \phi
$$

and

$$
i_{L_{f}}^{\prime}\left(\pi^{+}\right)=i_{L_{f}}^{\prime}\left(\pi^{-}\right)=\frac{V_{i}}{\omega L_{f}} .
$$

Normalising the initial conditions with respect to $i_{m}$ gives

$$
\begin{array}{r}
\frac{i_{L_{f}}}{i_{m}}\left(\pi^{+}\right)=\frac{\pi}{p}+\sin \phi \\
\frac{i_{L_{f}}^{\prime}}{i_{m}}\left(\pi^{+}\right)=\frac{1}{p}
\end{array}
$$

where

$$
p=\frac{\omega L_{f} i_{m}}{V_{i}} .
$$

Consequently, the coefficients $A$ and $B$ can be solved for and are equal to

$$
\begin{array}{r}
A=\frac{1}{q^{2}-1}\left(q \cos (q \pi) \cos \phi+\left(2 q^{2}-1\right) \sin (q \pi) \sin \phi\right) \\
+\frac{\pi}{p} \sin (q \pi)+\frac{1}{q p} \cos (q \pi), \quad q \neq 1 \\
B=\frac{1}{q^{2}-1}\left(-q \sin (q \pi) \cos \phi+\left(2 q^{2}-1\right) \cos (q \pi) \sin \phi\right) \\
+\frac{\pi}{p} \cos (q \pi)-\frac{1}{q p} \sin (q \pi), \quad q \neq 1 .
\end{array}
$$

Using Eqs. 22 and 32, the normalised voltage across the MOSFET with respect to the input voltage is equal to

$$
\begin{aligned}
\frac{v_{D S}(\omega t)}{V_{i}}= & 1-p \frac{d \frac{i_{L_{f}}}{i_{m}}(\omega t)}{d \omega t} \\
= & 1-A q p \cos (q \omega t)+B q p \sin (q \omega t) \\
& -\frac{q^{2} p}{q^{2}-1} \cos (\omega t+\phi), \quad q \neq 1 .
\end{aligned}
$$

For the specific case when $q=1$, which occurs when the circuit of the DC-feed inductance $L_{f}$ and the shunt capacitor $C_{1}$ is tuned to the switching frequency, the DC-feed inductor's current can be derived by substituting $q=1$ in Eq. 26 and resolving the differential equation with the initial conditions defined in Eqs. 30 and 31. The DC supply current is found by averaging the DC-feed inductor's current over the period 0 to $2 \pi$ which is also equal to average of the MOSFET's current

$$
\begin{aligned}
I_{o} & =\frac{1}{2 \pi} \int_{0}^{2 \pi} i_{L_{f}}(\omega t) d \omega t=\frac{1}{2 \pi} \int_{0}^{\pi} i_{D S}(\omega t) d \omega t \\
& =\frac{i_{m}}{2 \pi}\left(\frac{\pi^{2}}{2 p}-2 \cos \phi+\pi \sin \phi\right) .
\end{aligned}
$$

In an ideal Class $\mathrm{E}$ inverter there are no losses in the circuit, therefore all the power supplied by the power source is consumed in the reflected resistances $\left(R_{\mathrm{Ref}}\right.$ and $\left.r_{\mathrm{Ref}}\right)$ and the ESR of the primary coil $r_{P}$

$$
\begin{aligned}
P_{\text {in }} & =P_{\text {out }} \\
V_{i} I_{o}=\frac{v_{R}{ }^{2}}{2 R_{T}} & =\frac{1}{2} i_{m}{ }^{2} R_{T}
\end{aligned}
$$




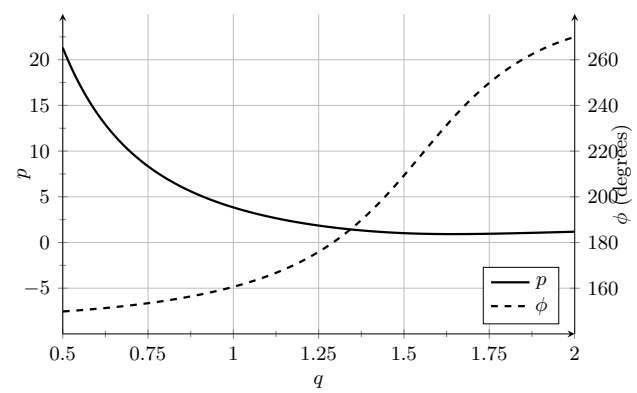

Fig. 3. The solved optimum parameters $p$ and $\phi$ versus $q$

where

$$
R_{T}=R_{\text {Ref }}+r_{\text {Ref }}+r_{P} .
$$

Rearranging the above equation and using Eq. 38 gives

$$
\frac{v_{R}}{V_{i}}=\frac{2 I_{o}}{i_{m}}=\frac{1}{\pi}\left(\frac{\pi^{2}}{2 p}-2 \cos \phi+\pi \sin \phi\right) .
$$

The normalised DC-feed inductor and the shunt capacitor with respect to the resistance $R_{T}$ can be derived using Eqs. 27, 32 and 41 and are given by

$$
\begin{gathered}
\frac{\omega L_{f}}{R_{T}}=p /\left(\frac{\pi}{2 p}-\frac{2}{\pi} \cos \phi+\sin \phi\right) \\
\omega R_{T} C_{1}=\frac{R_{T}}{q^{2} \omega L_{f}} .
\end{gathered}
$$

Substituting the zero-voltage switching (ZVS) and the zerovoltage derivative switching (ZVDS) conditions (i.e. $v_{D S}=0$ and $d v_{D S} / d \omega t=0$ at $\left.\omega t=2 \pi\right)$ in Eq. 36 results in the following two equations

$$
\begin{gathered}
1-A q p \cos (2 \pi q)+B q p \sin (2 \pi q)-\frac{q^{2} p}{q^{2}-1} \cos \phi=0 \\
A q^{2} p \sin (2 \pi q)+B q^{2} p \cos (2 \pi q)+\frac{q^{2} p}{q^{2}-1} \sin \phi=0
\end{gathered}
$$

Likewise, substituting the ZVS and ZVDS in Eq. ?? for the case when $q=1$

$$
\begin{gathered}
\frac{\pi p}{2} \sin \phi+2=0 \\
\frac{\pi p}{2} \cos \phi-2 p \sin \phi-\pi=0 .
\end{gathered}
$$

Equations 44 and 45 can be solved numerically as a function of $q$. Fig. 3 shows the solved values of $p$ and $\phi$. The value of the residual impedance is equal to

$$
X_{b}=\frac{v_{x m}}{i_{m}}=R_{T} \frac{v_{x m}}{v_{m}}
$$

where $v_{m}$ and $v_{x m}$ are the first two quadrature Fourier components and are equal to

$$
\begin{aligned}
v_{m} & =\frac{1}{\pi} \int_{\pi}^{2 \pi} v_{D S}(\omega t) \sin (\omega t+\phi) d \omega t \\
v_{x m} & =\frac{1}{\pi} \int_{\pi}^{2 \pi} v_{D S}(\omega t) \cos (\omega t+\phi) d \omega t .
\end{aligned}
$$

Therefore the normalised residual impedance is

$$
\frac{X_{b}}{R_{T}}=\frac{v_{x m}}{v_{m}}
$$

and the value of the capacitance $C$ is

$$
\omega C=\frac{1}{\omega L_{P}+X_{\operatorname{Ref}}+x_{\operatorname{Ref}}} .
$$

Therefore, the value of $C_{2}$ is

$$
C_{2}=\frac{1}{\frac{1}{C}+\omega X_{b}} .
$$

\section{B. Tuning and Optimisation}

From Eqs. 42, 43 and 51-53, the passive elements of the Class E inverter $L_{f}, C_{1}, C_{2}$ and $X_{b}$ are set for a specific reflected impedance at a certain switching frequency. The reflected impedance itself varies according to the distance, or the coupling coefficient, between the primary and secondary coils of the inductive link and the switching frequency. Any variations in the coupling coefficient will change the reflected impedance and alter the operation of the Class $E$ inverter from its optimal switching conditions. Therefore, it is necessary to recalculate the values of the passive elements that will allow the Class E inverter to operate at optimum switching conditions as the coupling coefficient changes. The aim of this section is to find the values of the switching frequency and the DC-feed inductance as the coupling coefficient varies while keeping the values of $C_{1}$ and $C_{2}$ fixed. To achieve this, we begin by substituting Eq. 42 in Eq. 43 which produces the following equation

$$
q^{2} p R_{t}(\omega, k) X_{C_{1}}-\left(\frac{\pi}{2 p}-\frac{2}{\pi} \cos \phi+\sin \phi\right)=0 .
$$

The residual impedance $X_{b}$ can be represented as the difference between the impedance of the primary coil, the imaginary components of the reflected impedances and the impedance of the series capacitors as follows

$$
X_{b}=X_{L_{P}}(\omega)+X_{\operatorname{Ref}}(\omega, k)+x_{\operatorname{Ref}}(\omega, k) .
$$

Substituting Eq. 55 in Eq. 51 results in the following equation

$$
\begin{array}{r}
X_{L_{P}}(\omega)+X_{\mathrm{Ref}}(\omega, k)+x_{\mathrm{Ref}}(\omega, k)- \\
X_{C_{2}}(\omega)-R_{T}(\omega, k) \frac{v_{x m}}{v_{m}}=0 .
\end{array}
$$

The optimum values of the switching frequency and the coupling coefficient can be found by finding the common roots for Eqs. 54 and 56 for specific values of $q, p$ and $\phi$. The optimum value of the DC-feed inductance can then be calculated by substituting the optimum values of the switching frequency and the coupling coefficient in Eq. 27. Fig. 4 shows a flowchart of the solution method to calculate the optimum values. The application of this solution method is demonstrated in a Class E inverter used as the primary coil driver in a WPT system. The complete specifications of the WPT system are shown in Table. I. The coupling coefficient varies from a minimum of 0.15 when the primary and secondary coils are misaligned to a maximum of 0.4 when the coils are aligned. Fig. 5 shows the solutions for the switching frequency and the DC-feed inductance against the coupling coefficient. The solution method was programmed in MATLAB using Chebfun [33], [34]. 


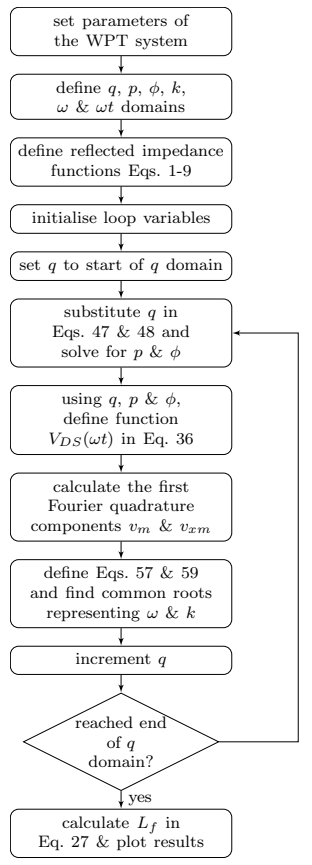

Fig. 4. Flowchart of the solution method to calculate the optimum values of the switching frequency and the DC-feed inductance as the coupling coefficient varies

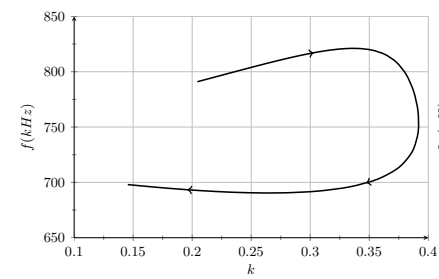

(a) Switching frequency

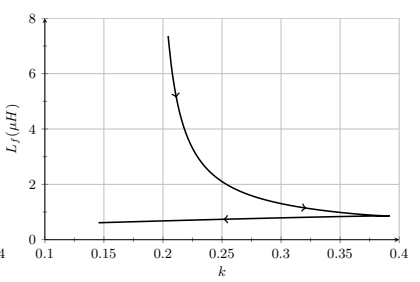

(b) DC-feed inductance
Fig. 5. The numerical solutions of Eqs. 54 and 56 for the WPT system specified in Table. I

The presented solution method is an iterative process that provides an exact solution. Other simpler design equations represented by second-order and third-order polynomial approximations [35] can be used but they may not be as accurate.

\section{SAturable Reactor as DC-Feed Inductance}

\section{A. Magnetic Cores}

As discussed in the previous section, tuning of the Class $\mathrm{E}$ inverter will be achieved by varying the DC-feed inductance and the switching frequency. In order to implement this tuning method during operation, the DC-feed inductance should be controlled by a voltage or current signal instead of performing mechanical adjustments. This can be achieved by using saturable reactors or magnetic amplifiers. The saturable reactor consists of a transformer wound on a magnetic core, the inductance of the primary windings can be altered by injecting DC magnetic flux into the core by means of a DC current applied to the secondary (control) windings. Detailed description on saturable reactors and their applications can be found in [36], [37].

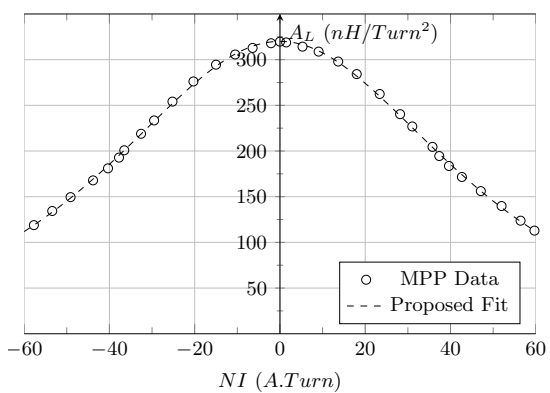

Fig. 6. MPP 550 data and proposed fit [38]

The magnetic core used in this paper is the 50202A MPP toroidal core from Magentics Inc. The core has an initial permeability of 550 , an effective cross-section area of $22.1 \mathrm{~mm}^{2}$ and an effective length of $50.9 \mathrm{~mm}$. Fig. 6 shows a plot of the DC bias performance of the MPP core used, which is obtained from the core manufacturer's datasheet [38]. The vertical axis represents the inductance factor in nanohenries per turn ${ }^{2}$, the horizontal axis represents the magnetoforce in ampere turns The following equation is proposed to provide an accurate fit for the data

$$
A_{L}=320 \operatorname{sech}(0.02854 N I) \times 10^{-9}
$$

where $A_{L}$ is the inductance factor, $N$ is the number of turns and $I$ is the DC current in the turns.

In general, the permeability of magnetic cores is not constant. It varies with frequency, the density of the magnetic flux and temperature. According to the manufacturer's specifications for the MPP core used [39], the permeability decreases to about $50 \%$ of its initial value at frequencies approaching $1 \mathrm{MHz}$. Conversely, the permeability increases to a peak of about $120 \%$ for AC flux density values of $0.1 \mathrm{~T}$. The relative permeability is 550 when the core's temperature is $20^{\circ} \mathrm{C}$ and increases by approximately $0.1 \%$ per degree Celsius. Since inductance is directly proportional to the permeability, the inductance of the primary windings can be multiplied by a factor representing the increase or decrease of the core's permeability. Therefore, by using Eq. 57, the overall DC-feed inductance, which consists of two identical series connected saturable reactors, as a function of the current in the control windings can be written as

$$
L_{f}=2 \times 320 r N_{p}^{2} \operatorname{sech}\left(0.02854 N_{c} I_{c}\right) \times 10^{-9}
$$

where $r$ is the reduction factor of the core's permeability, $N_{p}$ is the number of turns of the primary windings, $N_{c}$ is the number of turns in the control windings and $I_{c}$ is the DC controlling current.

Eq. 58 is used to provide an approximate value of the control current that is required to adjust the DC-feed inductance to retune the Class $\mathrm{E}$ inverter.

\section{EXPERIMENTAL RESULTS}

\section{A. System Specifications and Implementation}

A WPT system consisting of a Class E inverter and two magnetically coupled coils was built to verify the analysis 


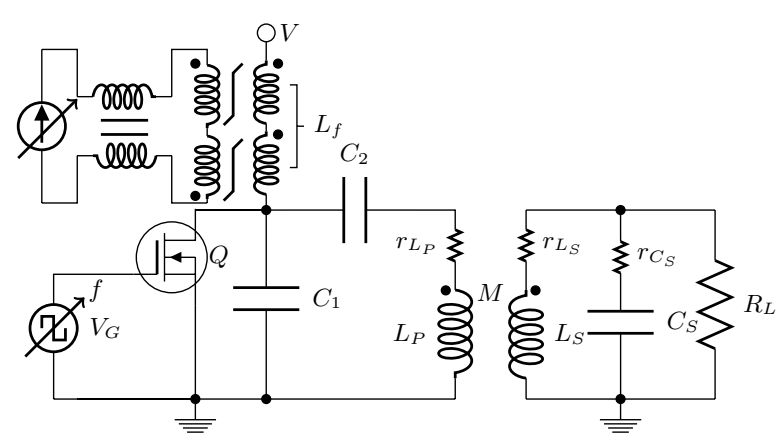

Fig. 7. Class E inverter with inductive link and controlled DC-finite inductance via a saturable reactor

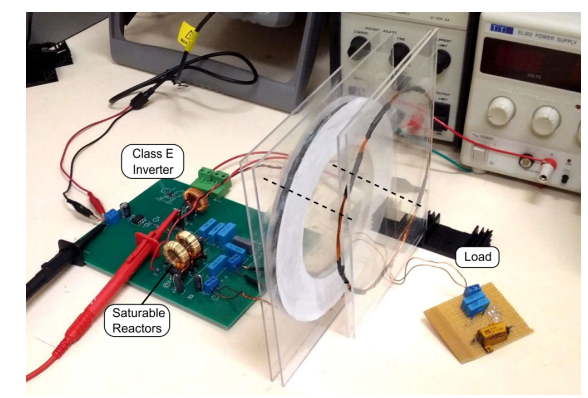

Fig. 8. The complete WPT system under test

described in the previous section, as well as to demonstrate the viability of the proposed tuning method to achieve optimised operation of the Class E inverter. The implemented circuit of the Class E inverter with the saturable reactors is shown in Fig. 7. The primary coil of the inductive link consists of two layers each having four turns of 18 AWG magnetic wire and a diameter of $16 \mathrm{~cm}$. The secondary coil consists of a single layer of four turns of 20 AWG magnetic wire with a diameter of $14 \mathrm{~cm}$. The DC-feed inductance consists of two saturable reactors connected in series as discussed in the previous section. The control windings are connected to a variable DC current source through a RFC choke. Based on initial measurements and experimental results, the reduction factor of the permeability of the saturable reactors' cores is assumed to be $65 \%$ at an operating temperature of $65^{\circ} \mathrm{C}$. The specifications and values of the inductive link, Class E inverter and the saturable reactors are summarised in Table. I. Fig. 8 shows the complete WPT system under test.

The primary and secondary coils of the inductive link are separated at a fixed distance of $2.5 \mathrm{~cm}$. The secondary coil is radially displaced from the primary coil causing the mutual inductance and the coupling coefficient to change, which leads to the reflected impedances to vary and therefore causing the Class E inverter to operate in a non-optimised switching condition. The DC current of the transformers and switching frequency are then altered to retune the Class E inverter.

\section{B. Electronic Tuning}

Depending on the coupling coefficient of the coupled coils, the amount of DC current injected in the secondary side of the transformers, along with the switching frequency, are
TABLE I

VALUES AND RANGES OF SEVERAL PARAMETERS OF THE Class E INVERTER AND THE INDUCTIVE LINK MEASURED AT $800 \mathrm{KHZ}$

\begin{tabular}{|c|c|c|}
\hline \multirow{10}{*}{ 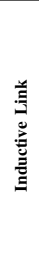 } & Component/Parameter & Value \\
\hline & $L_{P} / r_{P}$ & $5.76 \mu \mathrm{H} / 0.17 \Omega$ \\
\hline & $L_{S} / r_{S}$ & $6.69 \mu \mathrm{H} / 0.28 \Omega$ \\
\hline & $C_{S} / r_{C_{S}}$ & $5.92 \mathrm{nF} / 0.25 \Omega$ \\
\hline & Resonant Frequency $f_{O}$ & $800 \mathrm{kHz}$ \\
\hline & $R_{L}$ & $47 \Omega$ \\
\hline & Mutual Inductance $(M)$ Range & $1.37-2.23 \mu \mathrm{H}$ \\
\hline & Coupling Coefficient $(k)$ Range & $0.22-0.36$ \\
\hline & Coils Radial Displacement Range (d) & $0-2.5 \mathrm{~cm}$ \\
\hline & Coils Separation Dist. (h) & $2.5 \mathrm{~cm}$ \\
\hline \multirow{8}{*}{ 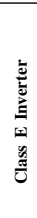 } & Component/Parameter & 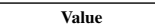 \\
\hline & MOSFET & STP40NF10 \\
\hline & Gate Driver & $\mathrm{TC} 1412$ \\
\hline & Input Voltage & $8 \mathrm{~V}$ \\
\hline & $C_{1}$ & $22 \mathrm{nF}$ \\
\hline & $C_{2}$ & $7.9 \mathrm{nF}$ \\
\hline & Switching Frequency Range $f_{\mathcal{S}}$ & $790-820 \mathrm{kHz}$ \\
\hline & Duty Cycle & $48 \%$ \\
\hline \multirow{8}{*}{ 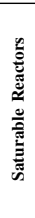 } & Component/Parameter & Value \\
\hline & Core Type & MPP 550 [38] \\
\hline & No. of Cores & 2 \\
\hline & Primary No. turns per core & 2.5 \\
\hline & Secondary No. turns per core & 70 \\
\hline & Permeability reduction factor $r$ & 0.65 \\
\hline & Control Current Range $I$ & $0.00-1.00 \mathrm{~A}$ \\
\hline & $L_{f}$ Range & $2.56-0.88 \mu \mathrm{H}$ \\
\hline
\end{tabular}

varied to retune the Class $\mathrm{E}$ inverter into operating at optimum switching conditions as the coils are radially displaced. Based on the solutions presented in Fig. 5, the switching frequency and the DC-feed inductance ranges chosen to be verified are $790 \mathrm{kHz}$ to $820 \mathrm{kHz}$ and $3.5 \mu \mathrm{H}$ to $1 \mu \mathrm{H}$ respectively. These chosen ranges allow the Class $\mathrm{E}$ inverter to operate near the resonant frequency of the inductive link which is $800 \mathrm{kHz}$. Fig. 9 shows the calculated DC-feed inductance and the switching frequencies as the coupling coefficient of the coil varies. The experimental results are in good agreement with the numerical solutions. Fig. 9c shows the measured power received at the load which is connected to the secondary coil of the inductive link, as well as the overall system efficiency which considers the inductive link losses, the Class E inverter losses, the MOSFET gate driver losses, the saturable reactors' core losses and the ohmic losses in the secondary windings of the saturable reactors. The received power is kept at a relatively constant level of $10 \mathrm{~W}$ throughout the displacement range with a peak occurring at a coupling coefficient of 0.3 . The overall efficiency is reduced when the coupled coils are closely aligned at higher coupling coefficients and is maintained at constant level as the coils are further displaced at lower coupling coefficients. Therefore, for the chosen values of the inductive link and the Class $\mathrm{E}$ inverter, the proposed tuning method is suited for reduced coupling coefficients and at large radial displacements of the coupled coils.

Fig. 10 shows the experimental waveforms of the MOSFET's drain voltage, the voltage of the load at the secondary coil, the voltage across one saturable reactor's control windings and the current of the saturable reactor's primary windings. In Fig. 10a, the inverter is operating at optimum switching conditions when the primary coils are aligned with each other. The coupling coefficient is 0.34 , the DC control current is $0.9 \mathrm{~A}$ and the switching frequency is $818 \mathrm{kHz}$. In Fig. 10b, the coils are radially displaced from each other by 


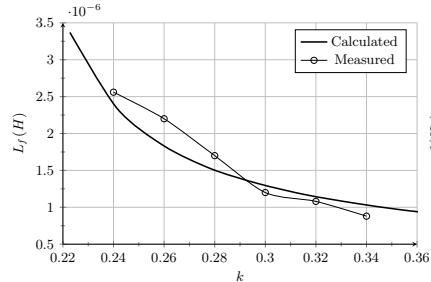

(a) DC-feed inductance $L_{f}$

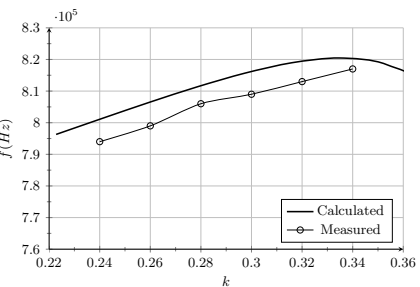

(b) Switching frequencies

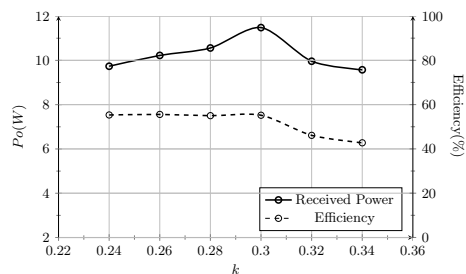

(c) Recieved power and overall system efficiency

Fig. 9. Calculated and measured parameters of the Class E inverter. Tuning is performed as the coupling coefficient varies
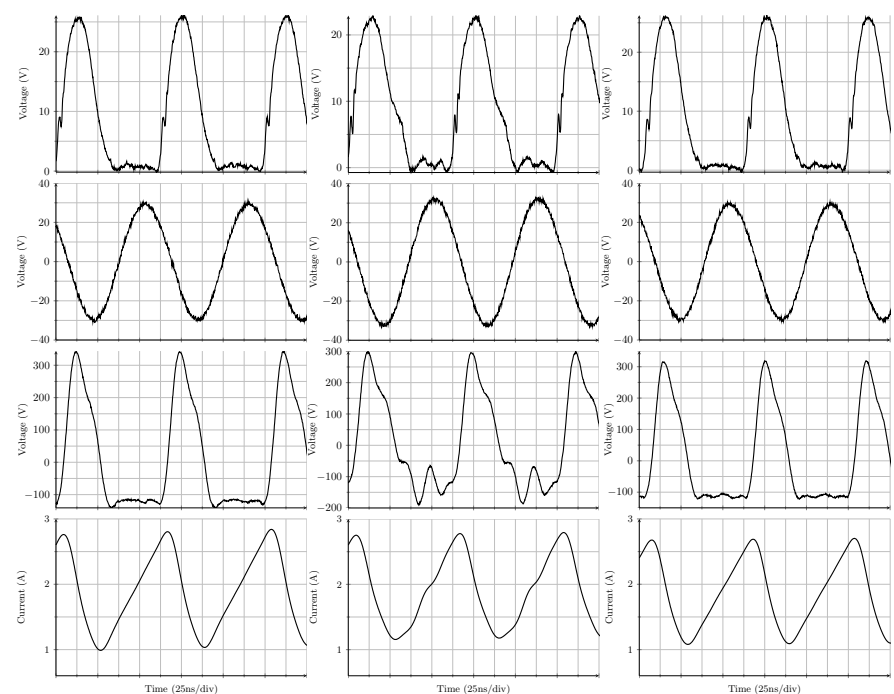

(a) Tuned optimum(b) Non-optimum switch-(c) Retuned optimum switching operation ating operation at $k=0.24$ switching operation at $k=0.34$

$k=0.24$

Fig. 10. Experimental waveforms of the implemented WPT system at different coupling coefficients demonstrating the proposed tuning method. First waveform is the MOSFET's drain voltage $V_{D S}$, second is the load voltage at the secondary coil, third is voltage across the control windings of one saturable reactor and the fourth is the current in the DC-feed inductance $I_{L_{f}}$ (primary windings of the saturable reactors)

a distance of $2.5 \mathrm{~cm}$ corresponding to a coupling coefficient of 0.24 , while maintaining the same DC control current and switching frequency. Since the coupling coefficient and the reflected impedances have now changed, the MOSFET switches $\mathrm{ON}$ at a non-zero voltage and therefore the inverter no longer operates at optimum switching conditions. In Fig. 10, the inverter is retuned and is now operating at optimum switching conditions by decreasing the DC control current to $0.05 \mathrm{~A}$ and the switching frequency to $795 \mathrm{kHz}$. Therefore, the proposed tuning method of controlling the DC-feed inductance and the switching frequency has been successfully verified. The new method allows the Class E inverter to operate safely and efficiently over a certain coil misalignment range.

\section{CONCLUSION AND FUtURE WORK}

A novel method has been presented to tune Class E inverters that are used as the primary coils driver in WPT systems based on resonant inductive coupling. By varying the inductance of the DC-feed inductor via a DC current source and the switching frequency, the Class $\mathrm{E}$ inverter can operate in an optimised switching condition achieving ZVS and ZVDS as the coupled coils of the inductive link are radially displaced. Mathematical analysis has been performed to derive the voltage and current relationships of the Class-E inverter based on a more accurate circuit representation of the inductive link. A numerical solution method is used to calculate the required DC-feed inductance and the switching frequency for tuning. The obtained experimental results are in good agreement with the numerical solutions and confirm the successful operation of the tuning method. It is also noted that proposed tuning method can be efficiently implemented electronically and may be applied to other WPT applications such as inductive charging for electric vehicles and mobile devices where displacements and coils misalignments are likely to occur.

Future work may include further investigation into other possible tuning methods such as adjusting the duty cycle of the MOSFET gate driving signal while maintaining the switching frequency at a constant value.

\section{REFERENCES}

[1] S. Raju, R. Wu, M. Chan, and C. P. Yue, "Modeling of mutual coupling between planar inductors in wireless power applications," IEEE Trans. Power Electron., vol. 29, no. 1, pp. 481-490, Jan 2014.

[2] J. P. C. Smeets, T. T. Overboom, J. W. Jansen, and E. A. Lomonova, "Comparison of position-independent contactless energy transfer systems," IEEE Trans. Power Electron., vol. 28, no. 4, pp. 2059-2067, Apr. 2013.

[3] K. Fotopoulou and B. Flynn, "Wireless power transfer in loosely coupled links: Coil misalignment model," IEEE Trans. Magn., vol. 47, no. 2, pp. 416-430, Feb. 2011.

[4] R. Jegadeesan, Y.-X. Guo, and M. Je, "Overcoming coil misalignment using magnetic fields of induced currents in wireless power transmission," in IEEE Int. Microwave Symp. Dig. (MTT), Jun. 2012, pp. 1-3.

[5] M. Pinuela, D. C. Yates, S. Lucyszyn, and P. D. Mitcheson, "Maximizing DC-to-load efficiency for inductive power transfer," IEEE Trans. Power Electron., vol. 28, no. 5, pp. 2437 - 2447, May 2013.

[6] T. P. Duong and J.-W. Lee, "Experimental results of high-efficiency resonant coupling wireless power transfer using a variable coupling method," IEEE Microw. Wireless Compon. Lett., vol. 21, no. 8, pp. 442 - 444, Aug. 2011.

[7] W. Zhong, C. K. Lee, and S. Hui, "General analysis on the use of Tesla's resonators in domino forms for wireless power transfer," IEEE Trans. Ind. Electron., vol. 60, no. 1, pp. 261 - 270, Jan. 2013.

[8] W. Zhong, X. Liu, and S. Y. R. Hui, "A novel single-layer winding array and receiver coil structure for contactless battery charging systems with free-positioning and localized charging features," IEEE Trans. Ind. Electron., vol. 58, no. 9, pp. 4136-4144, Sep. 2011.

[9] [Online]. Available: http://www.wirelesspowerconsortium.com.

[10] S. Aldhaher, P. C. K. Luk, and J. F. Whidborne, "Tuning Class inverters applied in inductive links using saturable reactors," IEEE Trans. Power Electron., vol. to be published, 2014.

[11] _ - "Wireless power transfer using Class E inverter with saturable DC-feed inductor," in Proc. IEEE Energy Convers. Congr. Expo., Sep. 2013, pp. 1902-1909.

[12] H. Wu, G. Covic, J. Boys, and D. Robertson, "A series-tuned inductivepower-transfer pickup with a controllable AC-voltage output," IEEE Trans. Power Electron., vol. 26, no. 1, pp. 98-109, Jan. 2011. 
[13] S.-H. Lee and R. Lorenz, "Development and validation of model for 95\%-efficiency $220-\mathrm{W}$ wireless power transfer over a 30 -cm air gap," IEEE Trans. Ind. Appl., vol. 47, no. 6, pp. 2495-2504, 2011.

[14] Z. Pantic and S. Lukic, "Wireless power transfer in loosely coupled links: Coil misalignment model," IEEE Trans. Power Electron., vol. 27, no. 11 , pp. 4503-4513, Nov. 2012.

[15] K. Schuylenbergh and R. Puers, Automatic link tuning. Springer Netherlands, 2009.

[16] N. O. Sokal and A. D. Sokal, "Class E-a new class of high-efficiency tuned single-ended switching power amplifiers," IEEE J. Solid-State Circuits, vol. 10, no. 3, pp. 168 - 176, Jun. 1975.

[17] Z. N. Low, R. A. Chinga, R. Tseng, and J. Lin, "Design and test of a high-power high-efficiency loosely coupled planar wireless power transfer system," IEEE Trans. Ind. Electron., vol. 56, no. 5, pp. 1801 1812, May 2009.

[18] G. A. Kendir, W. Liu, G. Wang, M. Sivaprakasam, R. Bashirullah, M. S. Humayun, and J. D. Weiland, "An optimal design methodology for inductive power link with class-E amplifier," IEEE Trans. Circuits Syst. I, Reg. Papers, vol. 52, no. 5, pp. 857 - 866, May 2005.

[19] M. V. Paemel, "High-efficiency transmission for medical implants," IEEE Solid State Circuits Mag., vol. 3, no. 1, pp. 47 - 59, Jan. 2011.

[20] E. M. Thomas, J. D. Heebl, C. Pfeiffer, and A. Grbic, "A power link study of wireless non-radiative power transfer systems using resonant shielded loops," IEEE Trans. Circuits Syst. I, Reg. Papers, vol. 59, no. 9, pp. $2125-2136$, Sept. 2012.

[21] J. J. Casanova, Z. N. Low, and J. Lin, "Design and optimization of a class-E amplifier for a loosely coupled planar wireless power system," IEEE Trans. Circuits Syst. II, Exp. Briefs, vol. 56, no. 11, pp. $830-$ 834, Nov. 2009.

[22] N. Kim, K. Kim, J. Choi, and C.-W. Kim, "Adaptive frequency with power-level tracking system for efficient magnetic resonance wireless power transfer," Electron. Lett., vol. 48, no. 8, pp. 452 - 454, 122012.

[23] H. Taghavi, B. Håkansson, and S. Reinfeldt, "Analysis and design of rf power and data link using amplitude modulation of class-E for a novel bone conduction implant," IEEE Trans. Biomed. Eng., vol. 59, no. 11, pp. 3050 - 3059, Nov. 2012.

[24] D. Murthy-Bellur, A. Bauer, W. Kerin, and M. Kazimierczuk, "Inverter using loosely coupled inductors for wireless power transfer," in IEEE 55th Int. Midwest Symp. on Circuits and Systems, 2012, pp. 1164-1167.

[25] A. Grebennikov and N. O. Sokal, Switchedmode RF Power Amplifiers. Oxford, UK: Newnes, 2007.

[26] M. Kazimierczuk and K. Puczko, "Exact analysis of Class-E tuned power amplifier at any Q and switch duty cycle," IEEE Trans. Circuits Syst., vol. 34, no. 2, pp. 149-159, Feb. 1987.

[27] M. K. Kazimierczuk, RF Power Amplifiers. Chichester, UK: John Wiley \& Sons, 2008.

[28] M. K. Kazimierczuk and D. Czarkowski, Resonant Power Converters, 2nd ed. New Jersey, USA: John Wiley \& Sons, 2011.

[29] D. Kessler and M. Kazimierczuk, "Power losses and efficiency of ClassE power amplifier at any duty ratio," IEEE Trans. Circuits Syst. I, Reg. Papers, vol. 51, no. 9, pp. 1675-1689, Sep. 2004.

[30] T. Suetsugu and M. Kazimierczuk, "Design procedure of Class-E amplifier for off-nominal operation at 50\% duty ratio," IEEE Trans. Circuits Syst. I, Reg. Papers, vol. 53, pp. 1468-1476, Jul. 2006.

[31] _ "Off-nominal operation of Class-E amplifier at any duty ratio," IEEE Trans. Circuits Syst. I, Reg. Papers, vol. 54, no. 6, pp. 1389-1397, Jun. 2007.

[32] — "Analysis and design of Class-E amplifier with shunt capacitance composed of nonlinear and linear capacitances," IEEE Trans. Circuits Syst. I, Reg. Papers, vol. 51, no. 7, pp. 1261-1268, Jul. 2004.

[33] Z. Battles and L. N. Trefethen, "An extension of Matlab to continuous functions and operators," SIAM J. Sci. Comp., 2004.

[34] L. N. Trefethen et al., Chebfun Version 4.2, The Chebfun Development Team, 2011, http://www.maths.ox.ac.uk/chebfun/.

[35] M. Acar, A. Annema, and B. Nauta, "Analytical design equations for Class-E power amplifiers," IEEE Trans. Circuits Syst. I, Reg. Papers, vol. 54, no. 12, pp. $2706-2717,2007$.

[36] M. K. Kazimierczuk, High-Frequency Magnetic Components. West Sussex, UK: John Wiley \& Sons, 2009.
[37] "Magnetic amplifiers: A rising star in naval electronics," Electronics Design and Development Division, Bureau of Ships, U. S. Navy, 1951.

[38] Magnetics Inc., MPP550 C055202A2 datasheet.

[39] MPP Material Curves, Magnetic Inc. [Online]. Available: http://www.mag-inc.com/products/powder-cores/mpp-cores/mppmaterial-curves

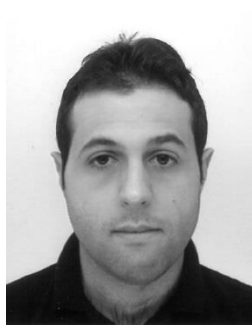

Samer Aldhaher received the B.Sc. degree in electrical engineering from the University of Jordan, Amman, Jordan in 2010. He is currently working towards the Ph.D. degree at Cranfield University, Bedford, UK.

His current research interests include the design of high frequency DC/AC inverters, wireless power transfer applications based on resonant inductive links and switched-mode circuits.

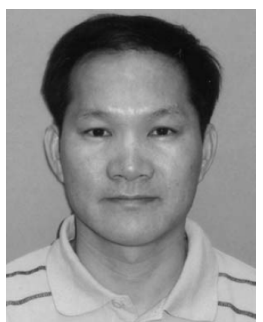

Patrick Chi-Kwong Luk (M'92-SM'08) is a native of Hong Kong. He received the High Diploma with merits (BSc) from Hong Kong Polytechnic University (PolyU), Hong Kong, in 1983, the M.Phil. degree from Sheffield University, U.K., in 1989, and the Ph.D. degree from the University of South Wales, U.K., in 1992, all in electrical engineering.

He started his career first in industry as an Assistant Engineer at GEC (H.K.) and then Application Engineer at Polytek Engineering Co. (H.K.). In 1986, he began his academic career as a Researcher at PolyU. Since 1988, he had held academic positions at the University of South Wales, U.K., Robert Gordon University, U.K., and University of Hertfordshire, U.K. In 2002, he joined Cranfield University, U.K., where he is currently Chair Professor in Electrical Engineering and Head of the Electric Power and Drives Group, School of Engineering. He is the Chairman of the IEEE UK\&RI Young Professionals and Professional Activities Affinity Groups, and is an Associate Editor for IEEE Transactions in Power Electronics. He has over 140 technical publications in electrical machines and drives. His main current research interests include electrical machines and converters for future transport and renewable energy applications. He has been invited as keynote speaker at international conferences.

A Senior Member of the IEEE, he won the 2011 IET Premium Award in Electric Power Applications.

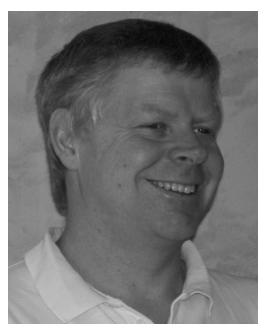

James F. Whidborne (M'95-SM'10) received the BA in engineering from Cambridge University and $\mathrm{MSc}$ and $\mathrm{PhD}$ in systems and control from University of Manchester Institute of Science and Technology (UMIST), UK

From 1991-1994, he was a research associate with the Department of Engineering, University of Leicester. From 1994-2003, he was a lecturer, then senior lecturer with the Department of Mechanical Engineering, Kings College London. He is currently a Reader and Head of the Dynamics Simulation and Control Group in the Department of Aerospace Engineering at Cranfield University, UK. His research interests are in the theory and application of advanced control, including multi-objective robust control design, fluid flow control, finite precision controller implementation problems, as well as flight control problems such as control and guidance of UAVs, controller reallocation and attitude control of VTOL aircraft.

$\mathrm{He}$ is a chartered engineer, a Member of the IET and a Senior Member of the IEEE. 